\title{
The Golgi apparatus is a primary site of intracellular damage after photosensitization with Rose Bengal acetate
}

\author{
C. Soldani, ${ }^{1}$ M.G. Bottone, ${ }^{1}$ A.C. Croce, ${ }^{2}$ A. Fraschini, ${ }^{1}$ G. Bottiroli, ${ }^{2}$ C. Pellicciari ${ }^{1,2}$ \\ ${ }^{1}$ Dipartimento di Biologia Animale and 'Istituto di Genetica Molecolare \\ (Sezione di Istochimica e Citometria) del CNR, University of Pavia, Italy
}

\begin{abstract}
(C)2004, European Journal of Histochemistry
The aim of the present investigation was to elucidate whether the Golgi apparatus undergoes photodamage following administration of the fluorogenic substrates Rose Bengal acetate (RBAc) and irradiation at the appropriate wavelength. Human HeLa cells were treated in culture and the changes in the organization of the Golgi apparatus were studied using fluorescence confocal microscopy and electron microscopy, after immunocytochemical labeling. To see whether the cytoskeletal components primarily involved in vescicle traffic (i.e., microtubules) might also be affected, experiments of tubulin immunolabeling were performed. After treatment with RBAc and irradiation, cells were allowed to grow in drug-free medium for different times. 24hr after irradiation, the cisternae of the Golgi apparatus became packed, and after 48-72 hr they appeared more fragmented and scattered throughout the cytoplasm; these changes in the organization of the Golgi cisternae were confirmed at electron microscopy. Interestingly enough, apoptosis was found to occur especially 48-72h after irradiation, and apoptotic cells exhibited a dramatic fragmentation of the Golgi membranes. The immunolabeling with anti-tubulin antibody showed that microtubules were also affected by irradiation in RBAc-treated cells.
\end{abstract}

Key words: Apoptosis, Confocal microscopy, Golgi apparatus, Photodynamic therapy, Rose Bengal acetate, Transmission electron microscopy

Correspondence: Carlo Pellicciari, Dipartimento di Biologia Animale, Università degli Studi di Pavia, Piazza Botta 10, 27100 Pavia, Italy.

E-mail: pelli@unipv.it

Paper accepted on October 22, 2004.

European Journal of Histochemistry 2004; vol. 48 issue 4 (Oct-Dec):443-448
T he photodynamic therapy (PDT) is a tumor eradication approach based on the action of photosensitizers (PS). PS are molecules which exhibit a preferential localization in tumor with respect to the normal tissues, and have a cytotoxic effect when excited by light at the appropriate wavelegths (Kennedy and Pottier, 1992; Dougherty et al., 1998; Gudgin Dickson et al., 2002). Unlike the common fluorochromes, PS have a high efficiency of intersystem crossing (i.e. the passage to the triplet state), in which the energy is preferentially dissipated through photochemical processes rather than by fluorescence emission. As a consequence, unstable, highly reactive oxydizing chemical species (singlet oxygen, free radicals or reactive oxygen species) are generated, which damage the cell molecular structures, and induce cell death by either necrosis or apoptosis (Kessel and Luo, 1999; Oleinick et al., 2002; Smetana and Hrkal, 2003).

PS can be chemically modified by introducing groups (such as acetate or phosphate groups) affecting the electron system of the fluorophore thus exerting a quenching effect on the photophysical (fluorescence emission) and photochemical (photosensitizing effect) properties of the modified PS. PS derivatives behave as fluorogenic substrates: once inside the cell, the added chemical groups are recognized and removed by cellular enzymes, so that the native chemical structure and its intrisic photophysical characteristics are restored (Bottiroli et al., 1997).

The xantene derivative, Rose Bengal is a very powerful PS producing singlet oxigen with high efficien$c y$, and its acetate derivative (RBAC) is a fluorogenic substrates whose application potential for PDT has already been demonstrated on cultured cell systems (Bottiroli et al., 1997; Croce et al., 2002). In fact, the presence of the acetate group was found to improve the intracellular influx of RBAc which has been suggested to enter the cell essentially through non receptor-mediated endocytotic mechanisms; previous evidence at fluorescence microscopy indi- 
cate that the drug first localizes in endosomes and then diffuses to the endoplasmic reticulum where esterases can restore the native photoactive molecule (Bottiroli et al., 1997). It has been hypothesized that the Golgi apparatus might also be involved, but a direct evidence for this event is still lacking (Bottiroli et al., 1997).

Thus, the aim of the present investigation was to elucidate whether the Golgi apparatus may actually undergo photodamage following RBAc administration and irradiation. To do this, human HeLa cells were treated in culture and the changes in the organization of the Golgi apparatus were studied using fluorescence confocal microscopy and electron microscopy, after immunocytochemical labeling. To see whether the cytoskeletal components primarily involved in vescicle traffic (i.e., microtubules) might also be affected, experiments of tubulin immunolabeling were performed.

\section{Materials and Methods}

\section{Cells and treatments}

Human HeLa cells (ATCC, Rockville, USA) were cultured in $75 \mathrm{~cm}^{2}$ flasks in Dulbecco's minimal essential medium supplemented with $10 \%$ fetal bovine serum, 1\% glutamine, $100 \mathrm{U}$ of penicillin and streptomycin (Celbio s.r.l., Milan, Italy) in 5\% $\mathrm{CO}_{2}$ humidified atmosphere. Twenty four hours before the experiments, cells were seeded on glass coverslips for fluorescence microscopy, or grown in either $25 \mathrm{~cm}^{2}$ or $75 \mathrm{~cm}^{2}$ plastic Petri dishes for electron microscopy.

Based on the results of previous loading experiments (not shown), cells were incubated for $60 \mathrm{~min}$ with $10^{-5} \mathrm{M}$ RBAc (Molecular Probes, Invitrogen S.r.l.); after incubation, the culture medium was replaced with Hank's Balanced Salt Solution (HBSS), previously allowed to get to equilibrium with $5 \% \mathrm{CO}_{2}$ humidified atmosphere, without phenol red, to avoid undesired photosensitizing effects. Irradiation (for a total dose of $1.6 \mathrm{~J} / \mathrm{cm}^{2}$ ) was performed, using as an innovative light source a light emitting diode (LED) at $530 \pm 15 \mathrm{~nm}$ (FRAEN Company, Milan, Italy). After irradiation cells were rinsed twice with HBSS, transferred to drug-free complete medium in which cells were allowed to recover for 24 to $72 \mathrm{~h}$.

As control samples, some cells were either incubated with RBAc, but not irradiated, or irradiated in the absence of RBAc incubation.

\section{Fluorescence microscopy}

The cells were fixed with $1 \%$ formaldehyde for 30 min at room temperature and then submitted to a dual-immunolabeling for the Golgi apparatus and microtubules. The Golgi membranes were labeled with an autoimmune serum (kind gift of dr. Claudia Alpini; dilution 1: 200 in PBS) recognizing a protein of 97 kDa (golgin-97: Griffith et al., 1997), revealed with a FITC-conjugated anti-human-IgG antibody (Molecular Probes, Invitrogen S.r.l.). The slides were then labeled with an anti- $\alpha$-tubulin antibody raised in mouse (diluted 1: 100 in PBS), finally revealed by an Alexa 594-conjugated anti-mouseIgG antibody (Molecular Probes, Invitrogen S.r.l.). All the incubations were performed for $60 \mathrm{~min}$ at room temperature, and the slides were counterstained for DNA with $0.1 \mu \mathrm{g} / \mathrm{mL}$ Hoechst 33258 and finally washed with PBS and mounted in Mowiol (Calbiochem, Inalco S.p.A., Milan, Italy).

\section{Confocal fluorescence microscopy}

For confocal laser scanning microscopy, we used a Leica TCS-SP system mounted on a Leica DMIRBE inverted microscope; for fluorescence excitation, an Ar/UV laser at $364 \mathrm{~nm}$ was used for Hoechst 33258, Ar/Vis laser at $488 \mathrm{~nm}$ for FITC and $\mathrm{He} / \mathrm{Ne}$ laser at 543 for Alexa 594. Spaced $(0.5$ $\mu \mathrm{m}$ ) optical sections were recorded using a $63 \times$ oil immersion objective. Images were collected in the 1024 x 1024 pixels format, stored on a magnetic mass memory and processed by the Leica Confocal Software.

\section{Transmission electron microscopy}

The cells were harvested by mild trypsinization ( $0.25 \%$ trypsin in PBS containing $0.05 \%$ EDTA), immediately fixed with $1 \%$ glutaraldehyde ( $1 \mathrm{~h}$ at $4^{\circ} \mathrm{C}$ ) in the culture medium and postfixed in $2 \%$ $\mathrm{OsO}_{4}$ for $\mathrm{I} \mathrm{h}$ at room temperature. The cell pellets were embedded in $2 \%$ agar in water, thoroughly rinsed with Sörensen buffer ( $\mathrm{pH}$ 7.2) and dehydrated in ethanol. Finally the cells were embedded in LR White resin and polymerized at $60^{\circ} \mathrm{C}$ for $24 \mathrm{~h}$.

To confirm the specific immunolabeling of the Golgi membranes, thin sections of mouse pancreas were used after fixation with $4 \%$ p-formaldehyde and $0.2 \%$ glutaraldehyde in $0.1 \mathrm{M}$ phosphate buffer $\mathrm{pH} 7.4$ medium for $2 \mathrm{~h}$ at $4^{\circ} \mathrm{C}$ (kind gift of dr. Manuela Malatesta). We decided to use pancreas sections because the acinar cells have a prominent Golgi apparatus which may be easily identified even 

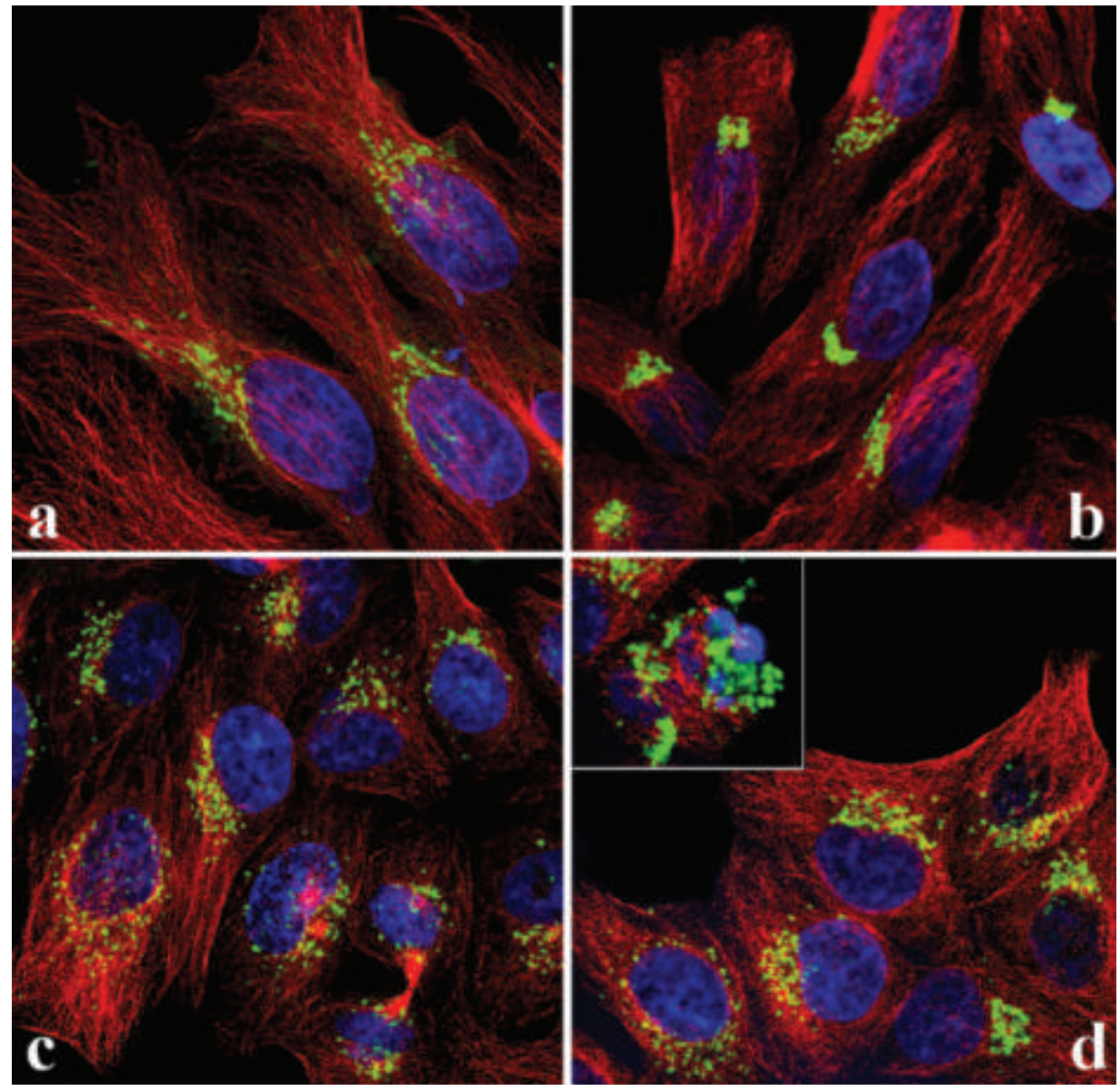

Figure 1. Dual-immulabeling of Golgi membranes (green fluorescence) and $\alpha$-tubulin (red fluorescence) in HeLa cells. a: untreated control; RBAc-treated and irradiated cells $24 \mathrm{~h} \mathrm{(b),} 48 \mathrm{~h}$ (c) and $72 \mathrm{~h}(\mathrm{~d})$ after irradiation. In the inset: apoptotic cell. Nuclear DNA was counterstained with Hoechst 33258 (blue fluorescence).

under the fixation conditions used (that are the most suitable for the immunolabeling procedures but provide a relatively poor morphological preservation). The thin sections were placed on nickel grids coated with a Formvar-carbon layer and then processed for the immunolabeling as described by Malatesta et al. (2003,a,b). Briefly, the sections were incubated with the autoimmune serum (dilution $1: 100$ with PBS) for $17 \mathrm{~h}$ at $4^{\circ} \mathrm{C}$ and then reacted for $20 \mathrm{~min}$ at room temperature with the secondary $12 \mathrm{~nm}$-gold-conjugated antibody (Jackson Immuno Research Europe Ltd, Soham, Cambridgeshire, UK) diluted 1:10 in PBS. Finally, the sections were rinsed and air-dried. As controls, some grids were treated with the incubation mixture without the primary antibody, and then processed as described above.

\section{Results}

In control cells, the Golgi apparatus has the typical ribbon-like distribution pattern around the nucleus after specific immunolabeling (Figure $1 a$ ).
After 60 min treatment with RBAc and irradiation, cells were allowed to grow in drug-free medium for different times: $24 \mathrm{~h}$ after irradiation, the cisternae of the Golgi apparatus became packed (Figure lb), and after 48-72 $\mathrm{h}$ they appeared more fragmented and scattered throughout the cytoplasm ( $1 \mathrm{c}$ and d).

The change in the organization of the Golgi cisternae was confirmed at electron microscopy, where they appeared more densely stacked (at $24 \mathrm{~h}$ postirradiation) than in untreated cells, to become progressively more dilated with large scattered vescicles at longer post-irradiation times (Figure $2 \mathrm{~b}, \mathrm{c}, \mathrm{e}$, $f)$. Moreover, the specificity of the autoimmune serum for the Golgi membranes was confirmed by gold-immunolabeling at electron microscopy (Figure 2a).

Interestingly enough, apoptosis was found to occur especially 48-72h after irradiation: apoptotic cells (about $20 \%$ at $72 \mathrm{~h}$, as estimated by morphological criteria) exhibited a dramatic fragmentation of the Golgi membranes (Figure $1 d$, inset and figure $2 d$ ).

The immunolabeling with anti-tubulin antibody 


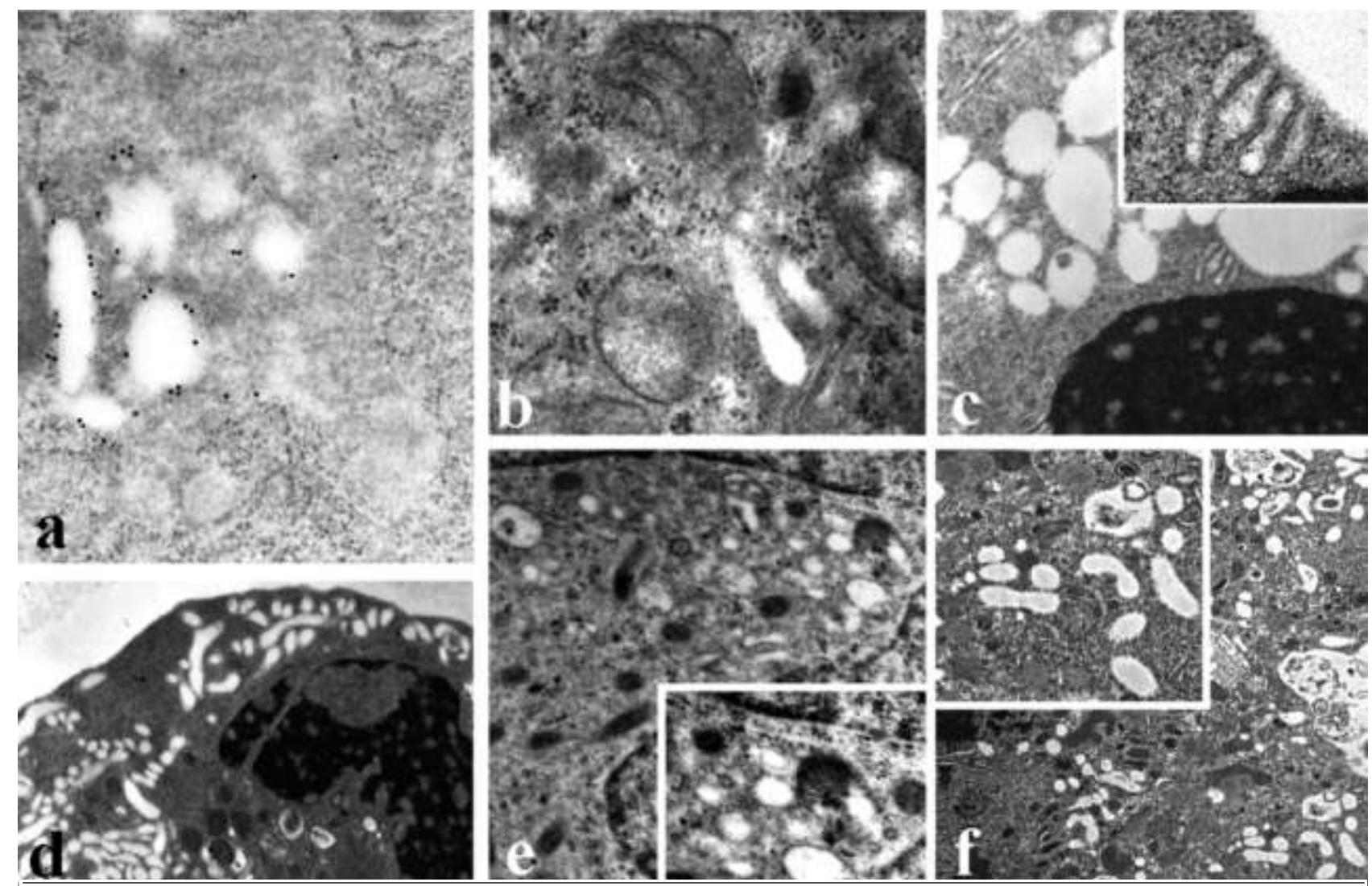

Figure 2. a: thin section of exocrine pancreas showing the specific gold-immunolabeling of the Golgi membranes; b: untreated HeLa cell, with short Golgi cisternae (arrow). In RBAc treated and irradiated cells, the Golgi cisternae are densely packed $24 \mathrm{~h}$ after irradiation (c), and progressively undergo fragmentation and dilation after $48 \mathrm{~h}$ and $72 \mathrm{~h}$ (e and f, respectively). In apoptotic cells (d), the fragmentation into large vesicles of endoplasmic and Golgi cisternae became dramatic.

showed that microtubules were also affected by irradiation in RBAc-treated cells: at 24h, microtubules aggregated into thick immunopositive bundles I Figure 1b), which underwent further reorganization at $48-72 \mathrm{~h}$ (Figure $1 \mathrm{c}, \mathrm{d}$ ), when microtubules were more irregularly dispersed than in control cells (Figure la).

No changes in the distribution of the Golgi cisternae or the microtubules were found in RBAc-treated, not irradiated samples, as well as in RBAcuntreated, light irradiated cells (not shown)

\section{Discussion}

PS molecules may enter the cells by different mechanisms, depending on their structural, physicochemical, charge and solubility properties, and they often exhibit selective specificity for different organelles (Peng et al., 1996; Dougherty et al., 1998). This specificity influences the intracellular localization of the PS which is crucial for the onset of the cytotoxic effect (Oleinick and Evans, 1998; Kessel e Luo, 1999; Rosenkranz et al., 2000; Oleinick et al, 2002), since PS-induced damages occur in close proximity of the excited molecules.

The mechanism proposed for the internalization of RBAc (i.e., endocytosis: Bottiroli et al., 1997) makes the involvement of the Golgi apparatus quite likely. In fact, as reported for other photosensitisers (Fabris et al., 2001; Teiten et al., 2003; Feofanov et al., 2004), our results demonstrate that also the RBAc-induced photodamage affects the Golgi apparatus as well as the microtubular cytoskeleton. This is not surprising, if one takes into account that a tight relationship esists between the microtubule system and the organization and dynamics of the Golgi apparatus: with its central location and pivotal role in membrane traffic, the Golgi apparatus is also in an ideal position to sense and integrate information about the state of the cell (Rios and Bornens, 2003).

Recent morphological studies have shown that the 
Golgi complex is fragmented during apoptosis (Sesso et al., 1999; Mancini et al., 2000; Machamer, 2003), and it has been suggested that the role played by this organelle in apoptosis could be dependent on the pro-apoptotic molecules (such as caspase2 and several death receptors) which are concentrated herein (Bennett et al., 1998; Mancini et al., 2000; Zhang et al., 2000). Cleavage of Golgi proteins, certainly contributes to Golgi disassembly in apoptotic cells which is required for packaging of the organelle into apoptotic blebs (Machamer, 2003).

PDT-induced cleavage of Golgi proteins could promote apoptotic signaling as well as the organelle fragmentation; in fact, during apoptosis, it has been reported that Golgi stacks disperse and disassemble into tubulo-vescicular clusters and determines the blockade of membrane traffic (Sesso et al, 1999; Lane et al., 2002), in a process that bears some similarity to the Golgi mitotic disassembly (Shorter and Warren, 2002). Chiu et al (2002) also demontrated that caspase-mediated proteolysis of key vesicle tethering factors ( $p 115$ ) contributes to Golgi breakdown during apoptosis and may act to propagate downstream apoptotic signals.

Nozawa et al. (2002) speculated that alterations of the Golgi complex may also facilitate the transport of death receptors from this organelle to the plasma membrane: this is an interesting hypothesis for antitumor PDT, since even a limited organelle damage by Golgi-specific PS could lead to massive apoptosis induction within the tumor mass, upon cell photosensitization.

\section{Acknowledgments}

This research was supported by MIUR (PRIN 2002, no 2002055584). We wish to thank $d r$. Manuela Malatesta (University of Urbino, Italy), who provided us with the thin sections of exocrine pancreas, and dr. Claudia Alpini (IRCCS San Matteo, Pavia, Italy), for the kind gift of the autoimmune serum recognizing a Golgi protein. Thanks are also due to Mrs Paola Veneroni for her skillful technical assistance in cell culture and treatment. CS was in receipt of a post-doc research contract (University of Pavia).

\section{References}

Bennett M, Macdonald K, Chan SW, Luzio JP, Simari R, Weissberg P. Cell surface trafficking of Fas: a rapid mechanism of p53-mediated apoptosis. Science 1998; 282:290-3

Bottiroli G, Croce AC, Balzarini P, Locatelli D, Baglioni P, Lo Nostro P, et al. Enzyme-assisted cell photodsensitization: a proposal for an efficient approach totumor therapy and diagnosis. The Rose Bengal fluorogenic substrate. Photochem Photobiol 1997; 66:374-83.

Chiu R, Novikov L, Mukherjee S, Shields D. A caspase cleavage fragment of p115 induces fragmentation of the Golgi apparatus and apoptosis. J Cell Biol 2002; 159:637-48.

Croce AC, Supino R, Lanza KS, Locatelli D, Baglioni P, Bottiroli G. Photosensitizers accumulation in spontaneous multidrug resistant cells: a comparative study with rhodamine 123 , rose bengal and photofrin. Photochem Photobiol Sci 2002; 1:71-8.

Dougherty TJ, Gomer CJ, Henderson BW, Jori G, Kessel D, Korbelik M, et al. Photodynamic therapy. Natl Cancer Inst 1998; 90:889-905.

English DS, Doyle RT, Petrich JW, Haydon PG. Subcellular distributions and excited-state processes of hypericin in neurons. Photochem Photobiol 1999; 69:301-05

Fabris C, Valduga G, Miotto G, Borsetto L, Jori G, Garbisa $S$, et al. Photosensitization with zinc (II) phthalocyanine as a switch in the decision between apoptosis and necrosis. Cancer Res 2001; 61:7495-500.

Feofanov A, Sharonov G, Grichine A, Karmakova T, Pljutinskaya A, Lebedeva $V$, et al. Comparative study of photodynamic properties of 13,15-N-cycloimide derivatives of chlorin p6. Photochem Photobiol 2004; 79:172-88

Griffith KJ, Chan EK, Lung CC, Hamel JC, Guo X, Miyachi K, et al. Molecular cloning of a novel 97-kd Golgi complex autoantigen associated with Sjogren's syndrome. Arthritis Rheum 1997;40:1693-702.

Gudgin Dickson EF, Goyan RL, Pottier RH. New directions in photodynamic therapy. Cell Mol Biol (Noisy-le-Grand) 2002; 48:939-54.

Kennedy JC, Pottier RH. Endogenpous proptoporphyrin IX, a clinically useful photosensitizer for photodynamic therapy. J Photochem Photobiol B Biol 1992; 14:275-92.

Kessel D, Luo Y. Photodynamic therapy: a mitochondrial inducer of apoptosis. Cell Death Differ 1999; 6:28-35.

Lane JD, Lucocq J, Pryde J, Barr FA, Woodman PG, Allan VJ, et al. Caspase-mediated cleavage of the stacking protein GRASP65 is required for Golgi fragmentation during apoptosis. J Cell Biol 2002; 156:495-509.

Machamer CE. Golgi disassembly in apoptosis: cause or effect? Trends Cell Biol 2003; 13:279-81.

Malatesta M, Baldelli B, Rossi L, Serafini S, Gazzanelli G. Fine distribution of CLOCK protein in hepatocytes of hibernating dormice. Eur $\mathrm{J}$ Histochem 2003a; 47:233-40.

Malatesta M, Biggiogera M, Manuali E, Baldelli B, Gazzanelli G. Fine structural analyses of pancreatic acinar cell nuclei from mice fed on genetically modified soybea Eur J Histochem 2003b; 47:385-8.

Mancini M, Machamer CE, Roy S, Nicholson DW, Thornberry NA, Casciola-Rosen LA, et al. Caspase-2 is localized at the Golgi complex and cleaves golgin-160 during apoptosis. J Cell Biol 2000; 149:603-12.

Nozawa K, Casiano CA, Hamel JC, Molinaro C, Fritzler MJ, Chan EK. Fragmentation of Golgi complex and Golgi autoantigens during apoptosis and necrosis. Arthritis Res 2002; 4:R3.

Oleinick NL, Evans HH. The photobiology of photodynamic therapy: cellular targets and mechanisms. Radiation Res 1998; 150:146-56,

Oleinick NL., Morris RL, Belichenko I. The role of apoptosis in response to photodynamic therapy: what, where, why, and how. Photochem Photobiol Sci 2002; 1:1-21.

Peng Q, Moan J, Nesland JM. Correlation of subcellular and intratumoral photosensitizer localization with ultrastrucutral features after photodynamic therapy. Ultrastruct Pathol 1996; 20:109-29.

Philpott KL, McCarthy MJ, Becker D, Gatchalian C, Rubin LL. Morphological and biochemical changes in neurons: apoptosis versus mitosis. Eur J Neurosci 1996; 8:1906-15. 


\section{Soldani et al.}

Rios RM, Bornens M. The Golgi apparatus at the cell center. Curr Opin Cell Biol 2003; 15:60-6

Rosenkranz AA, Jans DA, Sobolev AS. Targeted intracellular delivery of photosensitizers to nhance photodynamic efficiency. Immunol Cell Biol 2000; 78:452-64.

Sesso A, Fujiwara DT, Jaeger M, Jaeger R, Li TC, Monteiro M M, et al. Structural elements common to mitosis and apoptosis. Tissue Cell 1999; 31:357-71.

Shorter J, Warren G. Golgi architecture and inheritance. Annu Rev Cell Dev Biol 2002; 18:379-420.
Smetana K, Hrkal Z. Nucleoli in large (giant) bi-and multinucleate cells after apoptosis-inducing photodynamic treatment. Eur J Histochem, 2003; 47:39-44

Teiten $\mathrm{MH}$, Bezdetnaya L, Morliere $\mathrm{P}$, Santus $\mathrm{R}$, Guillemin F. Endoplasmic reticulum and Golgi apparatus are the preferential sites of Foscan localisation in cultured tumour cells. Br J Cancer 2003, 88:146-52

Zhang $M$, Zhang HQ, Xue SB. Effect of Bcl-2 and caspase-3 on calcium distribution in apoptosis of HL-60 cells. Cell Res 2000; 10: 213-20. 